\title{
Suppressive effects of microRNA-16 on the proliferation, invasion and metastasis of hepatocellular carcinoma cells
}

\author{
WEI-LU WU, WEI-YA WANG, WEN-QING YAO and GAN-DI LI \\ Department of Pathology, West China Hospital of Sichuan University, Chengdu 610041, P.R. China
}

Received April 12, 2015; Accepted September 22, 2015

DOI: $10.3892 / \mathrm{ijmm} .2015 .2379$

\begin{abstract}
R-16 is known to be abnormally expressed in hepatocellular carcinoma (HCC) cells, and the overexpression of miR-16 inhibits the proliferation, invasion and metastasis of various cancer cells. MicroRNAs (miRNAs or miRs) are closely related to the proliferation, invasion and metastasis of HCC. The present study aimed to explore the effects of miR-16 on the proliferation, invasion and metastasis of HCC cells, and to elucidate the mechanisms involved. A cell line with moderate levels of miR-16 expression was selected from the SMMC7721, HepG2, SK-Hep-1 and Huh-7 HCC cells and validated by reverse transcription-PCR (RT-PCR). The effects of miR-16 on HCC cell viability were determined by MTT assay; cell migration and invasion were determined by Transwell cell invasion assay, and apoptosis was determined by flow cytometery (FCM). Western blot analysis was used to measure the expression levels of the apoptosis-related proteins, Bax, Bcl-2, matrix metalloproteinase (MMP)-2, MMP-9, as well as to examine epithelial-mesenchymal transition (EMT), and E-cadherin, vimentin, and phosphatidylinositol 3-kinase (PI3K)/Akt signaling pathway-related protein expression. The mRNA expression levels of miR-16 were highest in the SMMC-7721 cells and lowest in the SK-Hep-1 and Huh-7 cells; moderate levels were observed in the HepG2 cells. The HepG2 cell line was selected as the cell line for use in the follow-up experiments, where we measured cell viability, and the expression of PI3k/Akt, Bax, Bcl-2, MMP-2 and MMP-9, and E-cadherin and vimentin. miR-16 overexpression significantly inhibited the proliferation, invasion and metastasis of the HepG2 cells, as shown by western blot anlaysis. This was achieved through the upregulation of Bax expression, the downregulation of Bcl-2 expression and the decrease in the expression of MMP-2 and MMP-9. In addition the epxression of E-cadherin increased
\end{abstract}

Correspondence to: Professor Gan-Di Li, Department of Pathology, West China Hospital of Sichuan University, 37 Guo Xue Alley, Chengdu, Sichuan 610041, P.R. China

E-mail: ligandi2015@126.com

Key words: microRNA-16, hepatocellular carcinoma, PI3K/Akt signaling pathway, E-cadherin expression and vimentin expression decreased. miR-16 overexpression inhibited PI3K expression and Akt phosphorylation. The results of this study suggest that the overexpression of miR-16 inhibits the proliferation, invasion and metastasis of HepG2 HCC cells, and that these effects are associated with the PI3K/ Akt signaling pathway.

\section{Introduction}

Of the malignant tumors which form in essential liver or intrahepatic bile duct, $90 \%$ are usually hepatocellular carcinoma (HCC) (1). HCC is the fifth most common solid tumor worldwide. China has the the highest incidence of liver cancer worldwide (2). The majority of patients are already in the advanced stages of the disease at the time of diagnsis $(1,3)$. At present, surgical resection and liver transplantation are the most effective treatment methods for HCC. However, clinical research has shown that $>50 \%$ of patients with HCC relapse following liver resection, an event which is related to the fact that HCC is a type of heterogeneous tumor with a high invasive- and metastatic capacity. Intrahepatic spread and extrahepatic invasion and metastasis are the leading causes of a poor prognosis for patients with $\mathrm{HCC}(4,5)$.

MicroRNAs (miRNAs or miRs) are a class of evolutionarily highly conserved endogenous, non-coding, small RNA molecules, which can combine with the mRNA 3'-UTR region of target genes, suppress the translation of target genes, and then inhibit the expression of target genes (6). miRNAs play an important role in various pathological and physiological processes, including carcinogenesis and cancer metastasis, and promote the proliferation and metastasis of malignant tumors, including HCC (7). The expression of miR-15a and miR-16-1 has been shown to be downregulated or lacking in the majority of patients with chronic lymphocytic leukemia (8). As regulatory factors, miRNAs can either play the role of proto-oncogenes or anti-oncogenes, affecting the biological characteristics of cells. Research has shown that the downregulated expression of miR-16 in HepG2, SK-HEP-1 and Huh7 HCC cells may be associated with the proliferation of HCC (9). miR-16 has been shown to suppress the proliferative activity of various cancer cells and can inhibit the invasion and metastasis of human glioma and colorectal cancer cells (10-12). Epithelialmesenchymal transition (EMT) is a key event in tumor invasion and metastasis. This process is not only involved in the disappearance of cancer cell polarity, but it is also closely related to 
the disappearance of the connection between cancer cells, the remodeling of the cytoskeleton, and changes to key signaling pathways. The induction of EMT and the abnormal expression of miRNAs are closely related to the occurrence, development, proliferation, migration and recurrence of tumors, including HCC (11-13).

The occurrence and development of HCC is a multi-step process and involves multiple different factors. It also involves the disorder of key signaling pathways that regulate various cellular processes, such as proliferation, apoptosis, invasion and metastasis. Previous research has confirmed that the abnormal activation of phosphatidylinositol 3-kinase (PI3K)/ protein kinase B (PKB or Alt) in HCC plays an important role in promoting cancer cell proliferation, apoptosis and metastasis (14). Bax, Bcl-2, and matrix metalloproteinases (MMPs), namely MMP-2 and MMP-9, are downstream molecules of the PI3K/AKT signaling pathway. Following activation, the PI3K /AKT pathway affects the expression of these downstream molecules, and then promotes the proliferation and invasion of HCC $(15,16)$. Tsai et al further confirmed that the activation of the PI3K/Akt signaling pathway was a key mechanism in the process of EMT, and that the suppressed activation of the pathway led to the reversal of EMT, the re-expression of E-cadherin and $\beta$-cadherin, and the reduction of the expression of the interstitial cell marker, vimentin (17). It has also been previously demonstrated that miR-16 regulates the expression of EMT-related proteins in U87 and U251 glioma cells through the Akt signaling pathway (12).

Based on the above-mentioned data, we formulated a hypothesis that miR-16 may inhibit the proliferation, invasion and metastasis of HCC cells, and that this effect may be mediated through the PI3K/Akt signaling pathway. Therefore, this study aimed to examine the effects of miR-16 on the expression of Akt signaling pathway-related proteins and on the proliferation, invasion and metastasis of HCC cells.

\section{Materials and methods}

Cell lines. Human SK-Hep-1 HCC cells were purchased from the Cell Bank of the Chinese Academy of Sciences (Beijing, China). The human HepG2 and SMCC-7721 cells were purchased from ATCC (Manassas, VA, USA). The Huh-7 HCC cells were purchased from Shanghai Bomaide Biological Technology. Co. Ltd. (Shanghai, China).

Reagents. MTT solution was obtained from Sigma Co. (St. Louis, MO, USA); rabbit anti-Bax (Cat no. 1063-1), antiBcl-2 (Cat no. 1017-1), anti-MMP-2 (Cat no. 1948-1) and anti-MMP-9 (Cat no. 1939-1) antibodies were purchased from Epitomics Co. (Burlingame, CA, USA); rabbit anti-PI3K (Cat no. 11889S), anti-Akt (Cat no. 2920) and antip-Akt (Cat no. 11962) antibodies were from Cell Signaling Techonology (Danvers, MA, USA); rabbit anti-E-cadherin (Cat no. 2707-1) and anti-vimentin (Cat no. 5409-1) antibodies were purchased from Abcam (Cambridge, MA, USA); $\beta$-actin antibody (Cat no. AA128), the Annexin V/PI double dye detection kit (Cat no. C1063), goat anti-rabbit and anti-mice antibody labeled with horseradish peroxidase (Cat no. A0208 and A0258) were all from the Beyotime Institute of Biotechnology (Jiangsu, China); Transwell Chambers were purchased from Corning Inc.
(Cat no. 3413, Corning, NY, USA); fetal bovine serum, DMEM culture medium and trypsin were all purchased from Gibco Life Technologies (Cat no. 10099-141 and 11965-092, Carlsbad, CA, USA); methyl thiazolyltetrazolium and crystal violet staining solution were from Sigma Co. (Cat no. M-2128 and 219215).

Instruments. The $\mathrm{CO}_{2}$ incubator was obtained from Thermo Scientific, Waltham, MA, USA; the super clean workbench was from Thermo Scientific; the inverted microscope was from Nikon, Tokyo, Japan; the flow cytometer was obtained from BD Biosciences (San Diego, CA, USA; the Mini dual vertical electrophoresis apparatus, the Mini transfer electrophoresis unit, and the ChemiDoc ${ }^{\mathrm{TM}}$ XRS Gel Imaging System were all from Bio-Rad, Hercules, CA, USA.

miRNA synthesis, preparation and storage. Synthetic miR-16 mimic, miR-16 inhibitor and mimic negative (NC) control, and inhibitor negative control were purchased from Guangzhou RiboBo Co., Ltd., (Guangzhou, China). Synthetic samples were first centrifuged $\left(10,000 \mathrm{rpm}, 25^{\circ} \mathrm{C}\right)$ then diluted per $5 \mathrm{nmol}$ miRNA, and subsequently added to $250 \mu \mathrm{l}$ diethylpyrocarbonate (DEPC)-treated water, which was added to $20 \mu \mathrm{M}$ mother liquor; the samples were repackaged and stored at $-20^{\circ} \mathrm{C}$. miR-16 mimic and $\mathrm{NC}$ mimic chemical synthesis were undertaken by Applied Biosystems, Foster City, CA, USA). The miR sequences were as follows: miR-16 mimic, 5'-UAGCAGCACGUAAAUAUUGGCGCCAAUAUUUAC GUGCUGCUAUU-3'; miR-16 mimic negative control, 5'-UUCUCCGAACGUGUCACGUTTACGUGACACGUUC GGAGAATT-3'; miR-16 inhibitor, 5'-CGCCAAUAUUUA CGUGCUGCUA-3'; and miR-16 inhibitor negative control, 5'-CAGUACUUUUGUGUAGUACAA-3'.

Transfection of cells with miRNAs. On the day prior to transfection, the cells were inoculated in medium without penicillin-streptomycin solution (6-well plates, 2,000 $\mu \mathrm{l}$ per well; increase or decrease in proportion). Cells were transfected when they reached a confluence of $30-50 \%$. The miRNAs were diluted with a moderate amount of serum-free Opti-MEM ${ }^{\circledR} \mathrm{I}$ culture medium (Cat. no. 31985-070; Invitrogen, Carlsbad, CA, USA) $(250 \mu \mathrm{l})$ by blending gently. Lipofectamine 2000 was diluted in Opti-MEM I by blending gently, followed by incubation for $5 \mathrm{~min}$ at room temperature. The diluted Lipofectamine 2000 (Cat. no. 13778150; Invitrogen) was gently mixed with the diluted miRNAs, and the mixture was incubated for 20-30 min at room temperature. Following the addition of the miRNA/Lipofectamine 2000 compound to the 6 -well plates, the plates were gently shaken. After $6 \mathrm{~h}$, the DMEM containing $10 \%$ serum was added, and the cells were cultivated at $37^{\circ} \mathrm{C}$ in a $\mathrm{CO}_{2}$ incubator for 24-72 h.

Reverse-transcription PCR (RT-PCR) validation of miR-16 expression in 4 different $H C C$ cell lines. Total RNA was extracted using TRIzol reagent (Invitrogen, Carlsbad, CA, USA), according to the manufacturer's instructions. The primer sequences were as follows: miR-16, 5'-TAGCAGCA CGTAAATATTGGCG-3'; and glyceraldehyde-3-phosphate dehydrogenase (GAPDH; internal control), 5'-TGACTTCAA CAGCGACACCCA-3'. RNA was reverse transcribed into cDNA and PCR amplification was carried out using a one-step 
RT-PCR kit (Cat. no. C81401180; Invitrogen). Approximately $5 \mu \mathrm{l}$ amplification product was used in the next step of $2 \%$ agarose gel detection. Following electrophoresis, bands were detected and images were acquired using an ultraviolet spectrophotometer (Quawell, San Jose, CA USA).

Determination of the cell proliferation rate by MTT assay. The HepG2 cells were inoculated in 96-well plates, with $100 \mu 1$ in each well and 4 plates for each group. When cell confluence reached 50\%, miR-16 mimic, miR-16 inhibitor and the corresponding negative control were transfected into the cells, and the transfection concentrations were 50 and $200 \mathrm{nmol}$, respectively. One day prior to transfection, the cells were digested and counted, and inoculated in 96-well plates. Following transfection for $48 \mathrm{~h}, 20 \mu 15 \mathrm{mg} / \mathrm{ml}$ MTT were added and followed by cultivation for $4 \mathrm{~h}$. After the nutrient solution was removed, $150 \mu \mathrm{l}$ DMSO were added to each well followed by shaking until the crystals had dissolved. The optical density (OD) was measured at $560 \mathrm{~nm}$ using an enzyme standard instrument, and the relative proliferation rate was calculated with $630 \mathrm{~nm}$ as the reference wavelength.

Analysis of cell migration. The HepG2 cells were inoculated in 96-well plates at $100 \mu \mathrm{l}$ in each well and 4 plates for each group. When the confluence of the cells reached 50\%, miR-16 mimic, miR-16 inhibitor and the corresponding negative controls were transfected into the cells, and the transfection concentrations were 50 and $200 \mathrm{nmol}$, respectively. On the day prior to transfection, the cells were digested and counted, and inoculated in 96-well plates. Following transfection for $48 \mathrm{~h}$, the cells were digested and added to a Transwell upper chamber, and the cells in the lower chamber continued to be cultured for a further $24 \mathrm{~h}$ in DMEM with 5\% fetal bovine serum. The Transwell chamber was subsequently removed and washed, and the cells were fixed with paraformaldehyde and stained with crystal violet. The number of migrated cells was counted in a total of 5 fields of view under an inverted optical microscope. The average number of cells per field of view was calculated, and this represented the migratory ability of the cells.

Analysis of cell invasion. The Matrigel was evenly spread on the microfilm of the Transwell chamber. The remaining steps were the same as those in described in 'RT-PCR validation of miR-16 expression in 4 different HCC cells lines'. The average number of HepG2 cells per field of view was calculated, which represented the invasive ability of the cells.

Detection of cell apoptosis. The HepG2 HCC cells were inoculated in 6-well plates. When the confluence of the cells reached $50 \%$, miR-16 mimic, miR-16 inhibitor and the corresponding negative controls were transfected into the cells, and the transfection concentrations were 50 and $200 \mathrm{nmol}$, respectively, with 3 wells/group. After $24 \mathrm{~h}$, the cells were digested and harvested, and then detected using a FACSCanto flow cytometry instrument (BD Biosciences) after being stained with Annexin V-FITC/PI for 30 min in the dark.

Western blot analysis. The HepG2 cells were inoculated in 96-well plates, $100 \mu \mathrm{l} /$ well and 4 plates/group. When the cell confluence reached $50 \%$, miR-16 mimic, miR-16 inhibitor

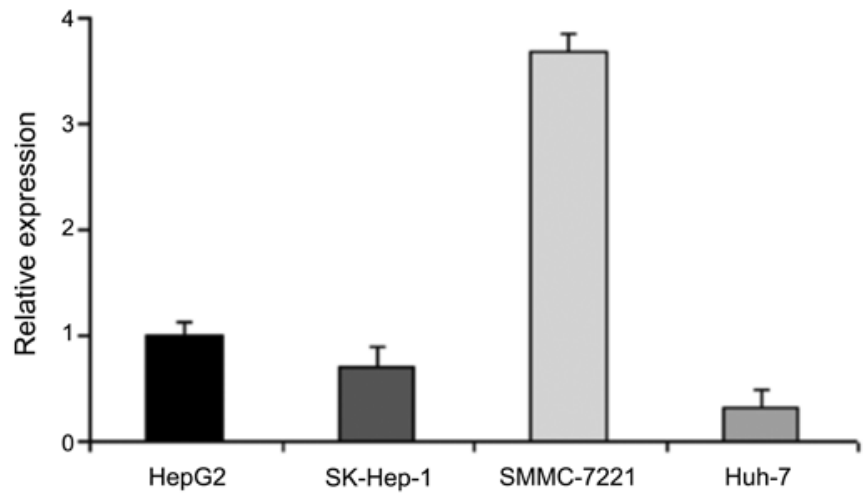

Figure 1. mRNA expression of miR-16 in 4 hepatocellular carcinoma (HCC) cell lines.

and the corresponding negative controls were transfected into the cells, and the transfection concentrations were 50 and $200 \mathrm{nmol}$, respectively. Following transfection for $48 \mathrm{~h}$, the cells were scraped and centrifuged $(10,000 \mathrm{rpm}$ for $10 \mathrm{~min}$ at $25^{\circ} \mathrm{C}$ ). Following the addition of the appropriate amount of RIPA lysis buffer, the cells were placed in a vortex meter and shaken for $30 \mathrm{sec}$. After $40 \mathrm{~min}$, they were centrifuged $\left(4^{\circ} \mathrm{C}\right.$, $10,000 \mathrm{rpm}$ ) for $10 \mathrm{~min}$, and the supernatant was then removed, and thus we obtained the total protein. The protein concentration was measured using a BCA kit. The proteins separated by SDS gel electrophoresis, and then transferred to the membrane using the wet transfer method. The membrane was immersed and incubated in primary antibody solution (Bax, Bcl-2, MMP 2, MMP 9, E-cadherin, vimentin, PI3K, $\beta$-actin, Akt and p-Akt) overnight at $4^{\circ} \mathrm{C}$. After being rinsed, the proteins were immersed and incubated in the secondary antibody solution (goat anti-rabbit and anti-mouse antibody labeled with horseradish peroxidase) at room temperature for 1-2 h. The membrane was removed and rinsed, followed by the addition of ECL solution, and were then examined using a gel imaging system. Each antibody gray value stripes were detected using Quantity One software.

Statistical analysis. Means and standard deviations (SD) were used to summarize continuous variables. To determine the differences between groups, the independent t-test and one-way ANOVA were used where appropriate. A two-sided P-value $<0.05$ was considered to indicate a statistically significant difference. All analyses were performed using SPSS software, version 17.0.

\section{Results}

Expression of the miR-16 gene in $4 \mathrm{HCC}$ cell lines. The results from RT-PCR revealed that of the 4 HCC cell lines, the mRNA expression level of miR-16 was highest in the SMMC-7721 cells and lowest in the SK-Hep-1 and Huh-7 cells; moderate expression levels were observed in the HepG2 cells (Fig. 1). Therefore, we selected HepG2 as the cell line for use in followup experiments.

Effect of miR-16 on HepG2 cell viability. The HepG2 cells were transfected with the miR-16 mimic and miR-16 inhibitor, 
Table I. Effect of miR-16 on HepG2 cell apoptosis.

\begin{tabular}{lcccc}
\hline Stage of apoptosis & Cont anti-miR & Cont miR & Anti-miR-16 & miR-16 \\
\hline Early apoptosis (\%) & $3.26 \pm 0.22$ & $0.84 \pm 0.13$ & $3.98 \pm 0.75$ & $17.24 \pm 2.56^{\mathrm{a}}$ \\
Late apoptosis (\%) & $0.7 \pm 0.16$ & $0.24 \pm 0.05$ & $1.76 \pm 0.58$ & $14.84 \pm 1.59^{\mathrm{a}}$ \\
\hline
\end{tabular}

${ }^{\text {a }}<0.01$ vs. control miR-16. Cont, negative control.

Table II. Effect of miR-16 on the migration and invasion of HepG2 cells.

\begin{tabular}{lcccr}
\hline Migration/invasion & Cont anti-miR & Cont miR & Anti-miR-16 & miR-16 \\
\hline Migration & $99.10 \pm 9.01$ & $99.23 \pm 7.93$ & $98.67 \pm 3.78$ & $66.76 \pm 6.39^{\mathrm{a}}$ \\
Invasion & $99.00 \pm 8.02$ & $99.09 \pm 9.26$ & $99.10 \pm 2.52$ & $67.35 \pm 5.73^{\mathrm{a}}$ \\
\hline
\end{tabular}

${ }^{\mathrm{a}} \mathrm{P}<0.01$ vs. control miR-16. Cont, negative control.

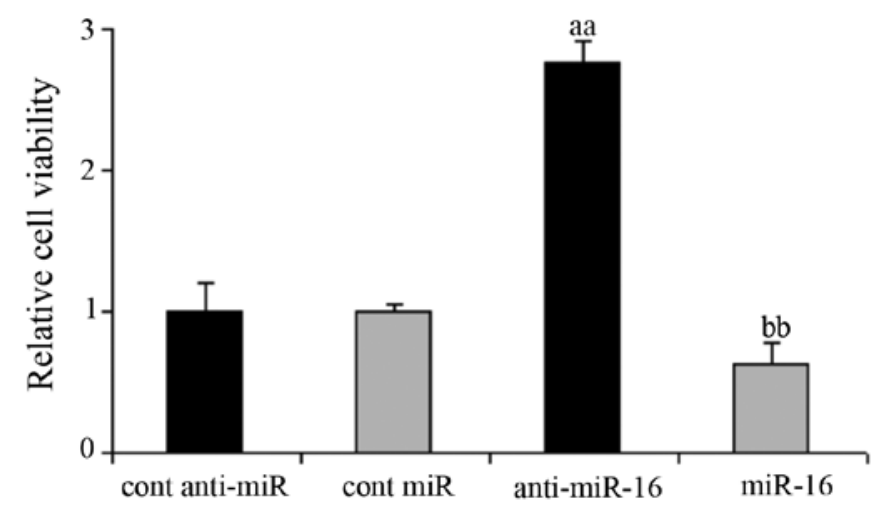

Figure 2. Effect of miR-16 on HepG2 cell viability. ${ }^{a} \mathrm{P}<0.05$ vs. negative control anti-miR-16 (cont miR); ${ }^{\text {bb }} \mathrm{P}<0.01$ vs. negative control miR-16 (cont anti-miR).

as well as the respective negative. The results of MTT assay revealed that compared with the negative control-transfected cells, the knockdown of miR-16 significantly increased HepG2 cell viability, whereas the overexpression of miR-16 inhibited HepG2 cell viability ( $\mathrm{P}<0.05$; Fig. 2).

Effect of miR-16 on HepG2 cell apoptosis. The results of Annexin V/PI flow cytometry revealed that compared with the negative control-transfected cells, the impact of miR-16 knockdown on HepG2 cell apoptosis was not significant. However, the overexpression of miR-16 significantly promoted HepG2 cell apoptosis $(\mathrm{P}<0.05$; Table I).

Effect of miR-16 on the migratory and invasive ability of Hep $G 2$ cells. The results of the migration and invasion assays revealed that the overexpression of miR-16 significantly reduced the number of HepG2 cells migrating through and invading the filter membrane $(\mathrm{P}<0.05)$. The impact of miR-16 knockdown on the migratory and invasive ability of the HepG2 cells was not significant (Table II).

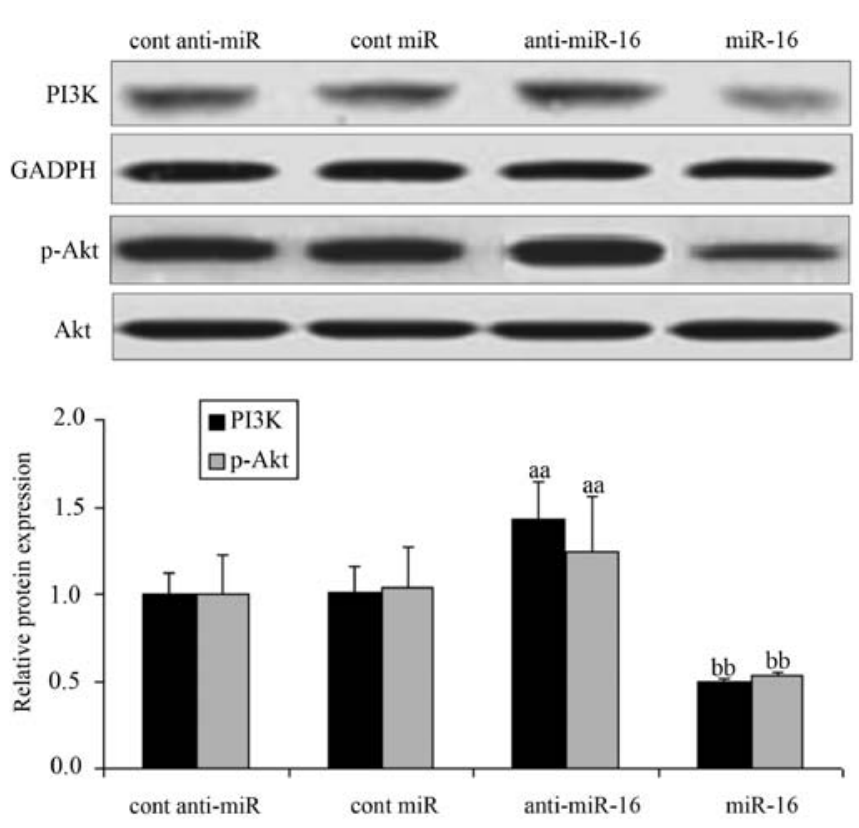

Figure 3. Effect of miR-16 on phosphatidylinositol 3-kinase (PI3K)/Akt signaling pathway-related protein expression in HepG2 cells. ${ }^{\text {aa }} \mathrm{P}<0.05$ vs. negative control anti-miR-16; ${ }^{\text {bb }} \mathrm{P}<0.01$ vs. negative control miR-16.

Effect of miR-16 on proteins related to the PI3K/Akt signaling pathway in HepG2 cells. The expression of PI3K/Akt pathwayrealted proteins in HepG2 cells was examined by western blot analysis. The expression level of PI3K and the Akt phosphorylation level in the cells transfected with the miR-16 inhibitor (anti-miR-16) were significantly increased compared with the levels in the negative control-transfected cells $(\mathrm{P}<0.05)$. Conversely, the expression level of PI3K and the Akt phosphorylation level were significantly decreased in the miR-16 mimic-transfected cells ( $\mathrm{P}<0.05$; Fig. 3$)$.

Effect of miR-16 on proteins related to apoptosis in HepG2 cells. Following transfection of the HepG2 cells with miR-16 

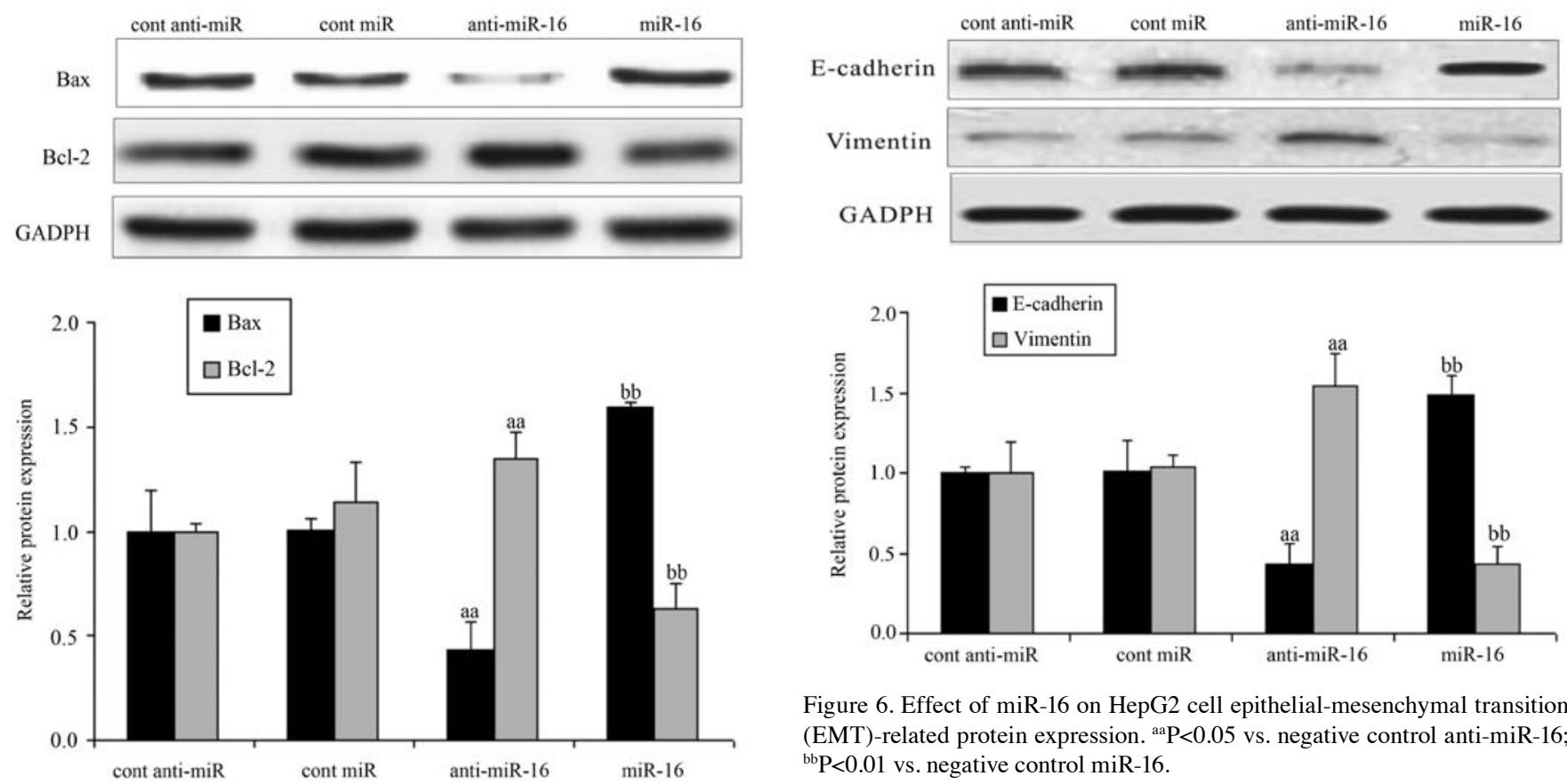

Figure 6. Effect of miR-16 on HepG2 cell epithelial-mesenchymal transition (EMT)-related protein expression. aa $\mathrm{P}<0.05$ vs. negative control anti-miR-16; ${ }^{\text {bb }} \mathrm{P}<0.01$ vs. negative control miR-16.

Figure 4. Effect of miR-16 on apoptosis-related proteins in HepG2 cells ${ }^{\text {aa }} \mathrm{P}<0.05$ vs. negative control anti-miR-16; ${ }^{\text {bb }} \mathrm{P}<0.01$ vs. negative control miR-16.
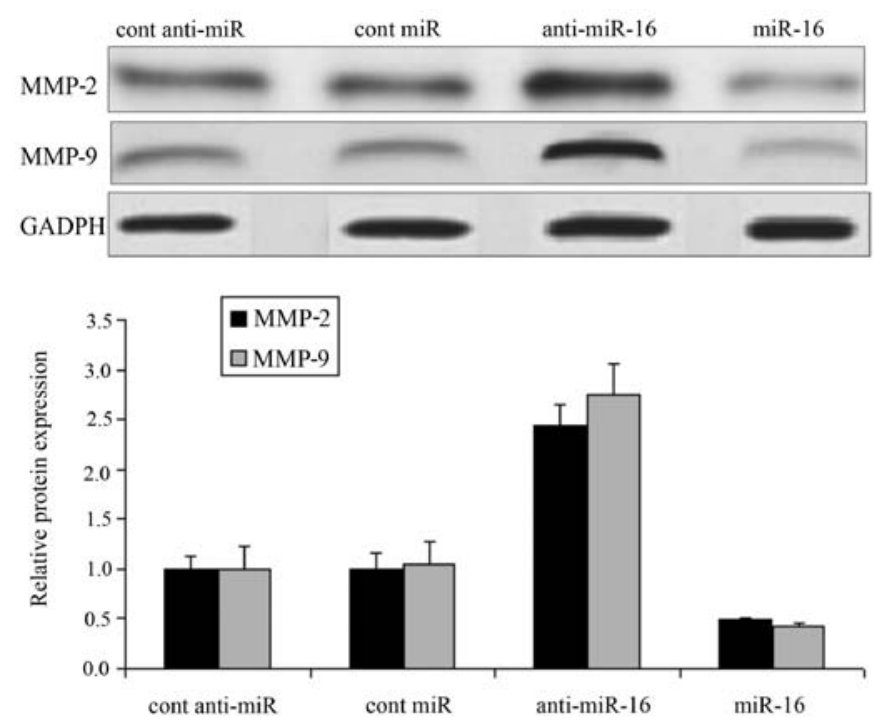

Figure 5. Effect of miR-16 on HepG2 cell matrix metalloproteinase (MMP) protein expression. ${ }^{\text {aa }} \mathrm{P}<0.05$ vs. negative control anti-miR-16; ${ }^{\text {bb }} \mathrm{P}<0.01$ vs. negative control miR-16.

mimic or miR-16 inhibitor, or the respective negative controls, we measured the expression levels of Bax and Bcl-2. Compared with the negative control-transfected cells, the expression level of Bax significantly decreased and that of Bcl-2 increased in the cells transfected with the miR-16 inhibitor (anti-miR-16) $(\mathrm{P}<0.05)$; however, the expression level of Bax significantly increased and that of Bcl-2 decreased in the cells transfected with the miR-16 mimic ( $\mathrm{P}<0.05$; Fig. 4).

Effect of miR-16 on MMP protein expression in HepG2 cells. Following transfection of the HepG2 cells with miR-16 mimic

or miR-16 inhibitor, or the respective negative controls, we measured the expression levels of MMP-2 and MMP-9 in the HepG2 cells. Compared with the negative control-transfected cells, the expression levels of MMP-2 and MMP-9 in the cells transfected with the miR-16 inhibitor (anti-miR-16) were significantly increased; however, the MMP-2 and MMP-9 expression levels were also markedly inhibited in the cells transfected with the miR-16 mimic $(\mathrm{P}<0.05$; Fig. 5).

Effect of miR-16 on EMT-related proteins in HepG2 cells. Following transfection of the HepG2 cells with miR-16 mimics or miR-16 inhibitor, or the respective negative controls, we measured the expression levels of E-cadherin and vimentin in the HepG2 cells. Compared with the negative control-transfected cells, the expression of E-cadherin was downregulated and that of vimentin was upregulated in the cells transfected with the miR-16 inhibitor (anti-miR-16) $(\mathrm{P}<0.05)$. In the cells transfected with the miR-16 mimic, the expression of E-cadherin was upregulated and that of vimentin was downregulated ( $\mathrm{P}<0.05$; Fig. 6$)$.

\section{Discussion}

miR-16 is an miRNA which exerts an antitumor effect and is closely associated with cancer cell proliferation and the development of malignant tumors. The results of the present study, revealed moderate mRNA expression levels of miR-16 in HepG2 cells, and thus this cell line was selected for use in follow-up experiments. We also observed the effects of miR-16 on the proliferation, invasion and metastasis of HepG2 HCC cells.

Bax and Bcl-2 are genes known to play a role in the regulation of apoptosis. Bax expression is downregulated in multiple tumors, including HCC tissue (18). Bcl-2 overexpression is clearly involved in the occurrence, development and metastasis of HCC cells (19). Studies have demonstrated that miRNAs are involved in cancer cell differentiation, apoptosis 
and proliferation, functioning as either tumor suppressor genes or proto-oncogenes $(19,20)$. miR-16 plays an extremely important biological role in the occurrence and development of tumors $(21,22)$. Bhattacharya et al reported that miR-16 and $-15 \mathrm{a}$ control Bmi-1 expression in ovarian cancer and that miR-15a and miR-16 overexpression inhibited the proliferation of ovarian carcinoma cells (23). Tang et al reported that miR-16 regulates the proliferation and apoptosis of ovarian epithelial carcinoma cells (24). In a previous study, microbubbles with rick miR-16 were subcutaneously injected into nude mice with prostate cancer and it was found that that miR-16 entered the cancer cells and inhibited the expression of Bcl-2 with fluorescent reporter gene expression (25). Our study also confirmed that miR-16 overexpression increased Bax expression and decreased Bcl-2 expression in HepG2 cells.

MMPs are a group of proteases, which play a key role in the degradation of the extracellular matrix, and in the invasion and metastasis of cancer cells. It has previously been demonstrated that MMPs play a crucial role in the process of $\mathrm{HepG} 2$ cell invasion (26). High expression levels of MMP-2 and MMP-9 have been shown to correlate with tumor size and the occurrence of lymphatic metastasis in HCC (27). Renjie and Haiqian (10) and Wang et al (12) reported that miR-16 overexpression inhibited the migration and invasion of glioma and pituitary cells. Yang et al that miR-16 inhibits glioma cell growth and invasion by suppressing Bcl-2 and the nuclear factor- $\mathrm{KB} 1 / \mathrm{MMP}-9$ pathway (25). In the present study, following transfection of HepG2 cells with miR-16 mimic or miR-16 inhibitor, we found that miR-16 overexpression decreased the expression levels of MMP-2 and MMP-9 and thus inhibited cell migration and invasion.

Previous research has indicated that EMT plays an important role in tumorigenesis, including local invasion, and transfer and diffusion through the circulatory system, and is related to the aggressiveness of cancer cells. The beginning of metastasis is said to occur when the EMT process commences in malignant cancer cells $(28,29)$. A marked characteristic of EMT is the downregulated expression of the intercellular adhesion molecule, E-cadherin, and the upregulated expression of a series of mesenchymal markers, including $\mathrm{N}$-cadherin, vimentin and fibronectin $(30,31)$. In HCC, the lack of E-cadherin expression is associated with tumor invasion, migration and a poor prognosis (32). It is considered that E-cadherin plays a role in the invasion ability of numerous cancer cells (33). Vimentin expression contributes to the enhanced invasion and metastasis in cancer $(32,34)$. Previous studies have reported that miR-16 influences cancer cell metastasis by affecting the process of EMT in colorectal cancer and pituitary tumor cells $(10,11)$. In the present study, we noted that miR-16 overexpression in HepG2 cells downregulated the expression of vimentin,and upregulated the expression of E-cadherin.

We also noted that miR-16 overexpression in HepG2 cells led to the significantly increased expression of PI3K and decreased levels of Akt phosphorylation. PI3K belongs to the phosphatidylinositol-dependent kinase family, and activates Akt through a series of signal transductions. Akt participates in various basic cellular processes, including cell proliferation, apoptosis, migration, invasion and EMT. In relation to HCC tissue, the abnormal activation of the PI3K/Akt pathway has been proven to be closely related to the prognosis of patients (35). It has also previously been demonstrated that PI3K/Akt activity is significantly decreased by the overexpression of miRNAs in HCC cells by treating HCC cells with miRNAs, thus inhibiting the proliferation, invasion and metastasis of HCC cells $(36,37)$.

Previous research has confirmed that mitochondrial dysfunction and deactivation of Akt may contribute to the apoptosis-inducing effects of carnosic acid in HepG2 cells (16). Mice MMP-2 gene knockout has been shown to affect the secretion of MMPs, which is mediated through the PI3K/Akt signaling pathway (38). In HepG2 cells, the downregulated expression of MMP-9, through the PI3K/Akt signaling pathway, has been shown to inhibit cell invasion (39). In the processes of cancer cell invasion and metastasis, it has been confirmed that EMT is associated with multiple signaling pathways. The activation of the Akt and Erk signaling pathways prompts the EMT process in cancer cells, providing cancer cells with the ability to survive (40). Wang et al (12) also found that miR-16 regulated EMT-related proteins in the glioma cell lines, U87 and U25, and that this involved the Akt signaling pathway; the decreased expression of E-cadherin and vimentin was also noted (12). Thus, miR-16 may also influence HepG2 cell proliferation, migration and invasion through the PI3K/Akt signaling pathway, and affect the expression of related proteins (12).

It must be noted that the experimental design of our current study had certain limitations. First, this study was designed in order to explore the influence of miR-16 on HepG2 cell proliferation, invasion, metastasis and apoptosisat the the protein level, not the genetic level. The synthesis of mRNA and protein should not be examined together as this would affect the results. Moreover, this study was an in vitro study. No in vivo experiments using animals were performed to observe the effects of miR-16 on the proliferation, invasion and metastasis of cancer cells from transplanted tumors. Thus, further in vivo animal studies are warranted to confirm our findings.

In conclusion, the findings of the present study demonstrated that miR-16 overexpression inhibited the proliferation, invasion and metastasis of HepG2 HCC cells, and that these effects involved the upregulation of Bax expression, the downregulation of Bcl-2 expression, and the decrease in the expression levels of MMP-2 and MMP-9. In addition, E-cadherin expression was increased and vimentin expression was decreased and this also involved the inhibition of PI3K expression and Akt phosphorylation.

\section{References}

1. Ichikawa T, Sano K and Morisaka H: Diagnosis of pathologically early HCC with EOB-MRI: Experiences and current consensus. Liver Cancer 3: 97-107, 2014.

2. Wei KR, Yu X, Zheng RS, Peng XB, Zhang SW, Ji MF, Liang ZH, Ou ZX and Chen WQ: Incidence and mortality of liver cancer in China, 2010. Chin J Cancer 33: 388-394, 2014.

3. Ringelhan M, O'Connor T, Protzer U and Heikenwalder M: The direct and indirect roles of HBV in liver cancer: prospective markers for HCC screening and potential therapeutic targets. J Pathol 235: 355-367, 2015.

4. de Lope CR, Tremosini S, Forner A, Reig M and Bruix J: Management of HCC. J Hepatol 56 (Suppl 1): S75-S87, 2012.

5. Finn RS: Advanced HCC: Emerging molecular therapies. Minerva Gastroenterol Dietol 58: 25-34, 2012.

6. Brodersen P, Sakvarelidze-Achard L, Bruun-Rasmussen M, Dunoyer P, Yamamoto YY, Sieburth L and Voinnet O: Widespread translational inhibition by plant miRNAs and siRNAs. Science 320: 1185-1190, 2008. 
7. Anwar SL and Lehmann U: MicroRNAs: emerging novel clinical biomarkers for hepatocellular carcinomas. J Clin Med 4: 1631-1650, 2015.

8. Calin GA, Cimmino A, Fabbri M, Ferracin M, Wojcik SE, Shimizu M, Taccioli C, Zanesi N, Garzon R, Aqeilan RI, et al: MiR-15a and miR-16-1 cluster functions in human leukemia. Proc Natl Acad Sci USA 105: 5166-5171, 2008.

9. Wu G, Yu F, Xiao Z, Xu K, Xu J, Tang W, Wang J and Song E: Hepatitis $B$ virus $X$ protein downregulates expression of the miR-16 family in malignant hepatocytes in vitro. Br J Cancer 105: 146-153, 2011.

10. Renjie W and Haiqian L: MiR-132, miR-15a and miR-16 synergistically inhibit pituitary tumor cell proliferation, invasion and migration by targeting Sox5. Cancer Lett 356B: 568-578, 2015.

11. Shi L, Jackstadt R, Siemens H, Li H, Kirchner T and Hermeking H: p53-induced miR-15a/16-1 and AP4 form a double-negative feedback loop to regulate epithelial-mesenchymal transition and metastasis in colorectal cancer. Cancer Res 74: 532-542, 2014.

12. Wang Q, Li X, Zhu Y and Yang P: MicroRNA-16 suppresses epithelial-mesenchymal transition-related gene expression in human glioma. Mol Med Rep 10: 3310-3314, 2014.

13. Zhang LY, Liu M, Li X and Tang H: miR-490-3p modulates cell growth and epithelial to mesenchymal transition of hepatocellular carcinoma cells by targeting endoplasmic reticulum-Golg intermediate compartment protein 3 (ERGIC3). J Biol Chem 288 : 4035-4047, 2013.

14. Dai XF, Ding J, Zhang RG, Ren JH, Ma CM and Wu G: Radiosensitivity enhancement of human hepatocellular carcinoma cell line SMMC-7721 by sorafenib through the MEK/ERK signal pathway. Int J Radiat Biol 89: 724-731, 2013.

15. Tian T, Nan KJ, Guo H, Wang WJ, Ruan ZP, Wang SH, Liang X and Lu CX: PTEN inhibits the migration and invasion of HepG2 cells by coordinately decreasing MMP expression via the PI3K/Akt pathway. Oncol Rep 23: 1593-1600, 2010.

16. Xiang Q, Ma Y, Dong J and Shen R: Carnosic acid induces apoptosis associated with mitochondrial dysfunction and $\mathrm{Akt}$ inactivation in HepG2 cells. Int J Food Sci Nutr 66: 76-84, 2015

17. Tsai JH, Hsu LS, Lin CL, Hong HM, Pan MH, Way TD and Chen WJ: 3,5,4'-Trimethoxystilbene, a natural methoxylated analog of resveratrol, inhibits breast cancer cell invasiveness by downregulation of PI3K/Akt and Wnt/ $\beta$-catenin signaling cascades and reversal of epithelial-mesenchymal transition. Toxicol Appl Pharmacol 272: 746-756, 2013.

18. Nagasawa T, Matsushima-Nishiwaki R, Toyoda H, Matsuura J, Kumada $\mathrm{T}$ and Kozawa O: Heat shock protein 20 (HSPB6) regulates apoptosis in human hepatocellular carcinoma cells: Direct association with Bax. Oncol Rep 32: 1291-1295, 2014.

19. Zhao N, Sun BC, Zhao XL, Wang Y, Sun HZ, Dong XY, Meng J and Gu Q: Changes in microRNAs associated with Twist-1 and Bcl-2 overexpression identify signaling pathways. Exp Mol Pathol 99: 524-532, 2015

20. Ambros V: The functions of animal microRNAs. Nature 431 350-355, 2004

21. Chamorro-Jorganes A, Araldi E, Penalva LO, Sandhu D, Fernández-Hernando $\mathrm{C}$ and Suárez Y: MicroRNA-16 and microRNA-424 regulate cell-autonomous angiogenic functions in endothelial cells via targeting vascular endothelial growth factor receptor-2 and fibroblast growth factor receptor-1. Arterioscler Thromb Vasc Biol 31: 2595-2606, 2011.

22. Goretti E, Rolland-Turner M, Léonard F, Zhang L, Wagner DR and Devaux Y: MicroRNA-16 affects key functions of human endothelial progenitor cells. J Leukoc Biol 93: 645-655, 2013.

23. Bhattacharya R, Nicoloso M, Arvizo R, Wang E, Cortez A Rossi S, Calin GA and Mukherjee P: MiR-15a and MiR-16 control Bmi-1 expression in ovarian cancer. Cancer Res 69 : 9090-9095, 2009.

24. Tang R, Cui ZM and Lou YH: MicroRNA-16 regulates the proliferation, invasion and apoptosis of ovarian epithelial carcinoma cells in vitro. Zhonghua Fu Chan Ke Za Zhi 47: 846-850, 2012 (In Chinese).
25. Yang TQ, Lu XJ, Wu TF, Ding DD, Zhao ZH, Chen GL, Xie XS, Li B, Wei YX, Guo LC, et al: MicroRNA-16 inhibits glioma cell growth and invasion through suppression of BCL2 and the nuclear factor- $\kappa \mathrm{B} 1 / \mathrm{MMP} 9$ signaling pathway. Cancer Sci 105: 265-271, 2014.

26. Ordoñez R, Carbajo-Pescador S, Prieto-Dominguez N, GarcíaPalomo A, González-Gallego J and Mauriz JL: Inhibition of matrix metalloproteinase-9 and nuclear factor kappa B contribute to melatonin prevention of motility and invasiveness in HepG2 liver cancer cells. J Pineal Res 56: 20-30, 2014.

27. Zhang Y, Shen Y, Cao B, Yan A and Ji H: Elevated expression levels of androgen receptors and matrix metalloproteinase-2 and -9 in 30 cases of hepatocellular carcinoma compared with adjacent tissues as predictors of cancer invasion and staging. Exp Ther Med 9: 905-908, 2015.

28. Ogunwobi $\mathrm{OO}$ and Liu C: Therapeutic and prognostic importance of epithelial-mesenchymal transition in liver cancers: Insights from experimental models. Crit Rev Oncol Hematol 83: 319-328, 2012.

29. Yang JD, Nakamura I and Roberts LR: The tumor microenvironment in hepatocellular carcinoma: Current status and therapeutic targets. Semin Cancer Biol 21: 35-43, 2011.

30. Hou KZ, Fu ZQ and Gong H: Chemokine ligand 20 enhances progression of hepatocellular carcinoma via epithelial-mesenchymal transition. World J Gastroenterol 21: 475-483, 2015.

31. Yamada S, Okumura N, Wei L, Fuchs BC, Fujii T, Sugimoto H, Nomoto S, Takeda S, Tanabe KK and Kodera Y: Epithelial to mesenchymal transition is associated with shorter disease-free survival in hepatocellular carcinoma. Ann Surg Oncol 21: 3882-3890, 2014

32. Zhai X, Zhu H, Wang W, Zhang S, Zhang Y and Mao G: Abnormal expression of EMT-related proteins, S100A4, vimentin and E-cadherin, is correlated with clinicopathological features and prognosis in HCC. Med Oncol 31: 970, 2014.

33. Wang $\mathrm{H}$ and Zhou Q: E-cadherin/beta-catenin and the invasion and metastasis of lung cancer. Zhongguo Fei Ai Za Zhi 13: 254-259, 2010 (In Chinese).

34. Wang TH, Lin YS, Chen Y, Yeh CT, Huang YL, Hsieh TH, Shieh TM, Hsueh C and Chen TC: Long non-coding RNA AOC4P suppresses hepatocellular carcinoma metastasis by enhancing vimentin degradation and inhibiting epithelial-mesenchymal transition. Oncotarget 6: 23342-23357, 2015.

35. Zhou Q, Lui VW and Yeo W: Targeting the PI3K/Akt/mTOR pathway in hepatocellular carcinoma. Future Oncol 7: 1149-1167, 2011.

36. Lang Q and Ling C: MiR-124 suppresses cell proliferation in hepatocellular carcinoma by targeting PIK3CA. Biochem Biophys Res Commun 426: 247-252, 2012.

37. Yan H, Wang S, Yu H, Zhu J and Chen C: Molecular pathways and functional analysis of miRNA expression associated with paclitaxel-induced apoptosis in hepatocellular carcinoma cells. Pharmacology 92: 167-174, 2013.

38. Chetty C, Lakka SS, Bhoopathi P and Rao JS: MMP-2 alters VEGF expression via alphaVbeta3 integrin-mediated PI3K/AKT signaling in A549 lung cancer cells. Int J Cancer 127: 1081-1095, 2010.

39. Shan RF, Zhou YF, Peng AF and Jie ZG: Inhibition of Aurora-B suppresses HepG2 cell invasion and migration via the PI3K/Akt/ NF- $\mathrm{BB}$ signaling pathway in vitro. Exp Ther Med 8: 1005-1009, 2014.

40. Xu L, Zhang Y, Wang H, Zhang G, Ding Y and Zhao L: Tumor suppressor miR-1 restrains epithelial-mesenchymal transition and metastasis of colorectal carcinoma via the MAPK and PI3K/AKT pathway. J Transl Med 12: 244, 2014. 